\title{
Biodanza: revisión sistemática sobre los beneficios de una práctica emergente en la promoción de la salud y el bienestar \\ Biodanza: systematic review of the benefits of an emerging practice in the promotion of health and well-being \\ Claudia Altamirano Quevedo, Estefanía Castillo Viera, Iván Rodríguez Pascual \\ Universidad de Huelva (España)
}

\begin{abstract}
Resumen. La Biodanza es una disciplina emergente que parece estar relacionada con la salud y el bienestar. Basada en un sistema integrador de disciplinas como la danza, la música y sobre todo la expresión corporal, que permite a los participantes adaptar su nivel inicial y sus capacidades. Para conocer los beneficios que aporta la práctica de biodanza se hace necesario la revisión sistemática de la literatura científica. El objetivo fue realizar una revisión sistemática de la literatura científica sobre biodanza y su relación con la salud. Método: se identificaron los estudios en las bases de datos Web of Science y Scopus hasta enero de 2020. Se seleccionaron las investigaciones de diseño cuantitativo, publicadas en inglés o castellano. Tras el cribado, se identificaron nueve artículos que cumplían los criterios de inclusión. Resultados: la biodanza muestra resultados positivos sobre la salud de mujeres con fibromialgia, así como en población general y niños. Las variables analizadas fundamentalmente fueron depresión, ansiedad, calidad del sueño, manejo del dolor, o inteligencia emocional. Conclusiones: los estudios muestran una relación positiva entre los practicantes de biodanza y algunos parámetros de salud, especialmente variables referidas al bienestar, así como una clara utilidad en el trabajo comunitario y la promoción de la salud. Los estudios son escasos y las muestras no son aleatorias, por lo que no se pueden generalizar fácilmente los resultados.

Palabras clave: salud, danza, calidad de vida, expresión corporal, actividad física.
\end{abstract}

\begin{abstract}
Biodanza is an emerging discipline apparently related to health and well-being. It is based on an system integrating disciplines such as dance, music, and, particularly, body expression, which allows participants to adapt their initial level and abilities. In order to know the benefits of the practice of biodanza, it is necessary to systematically review the scientific literature related to it. Objective: this article aims to systematically review the benefits of biodanza on health. Method: the studies were identified in the databases until December 2018. Quantitative design research, published both in English and Spanish, were selected. Nine articles meeting the inclusion criteria were identified. Results: biodanza shows positive results on the health of women with fibromyalgia, as well as in the general population and children. Depression, anxiety, quality of sleep, or emotional intelligence were among the health variables analyzed. Conclusions: the studies show a positive relationship between biodanza practitioners and some health parameters, especially wellbeing variables. Biodanza seems to have also a clear utility in community work and health promotion. The studies are still scarce and the samples are not random, so the results cannot be easily generalized.
\end{abstract}

Keywords: health, dance, quality of life, body techniques, body language, physical activity.

\section{Introducción}

En los últimos años se ha incrementado el número de estudios que investigan las disciplinas y actividades que contribuyen al bienestar subjetivo de las personas y, dentro de ellas, aquellas actividades expresivas que reconocen el cuerpo e integran la danza como recurso para la salud (Padilla y Coterón, 2013). En esta línea se encuentra la Biodanza como intervención psico-física destinada a mejorar la salud (Castañeda, 2009; Giannino, Gianelli y Mengarelli, 2015), siendo un método de facilitación de expresión del potencial humano mediante la música, la danza, las vivencias integradoras y la comunicación en grupo (Cavalcante y Wagner, 2015; León y González-Martí, 2017). La Biodanza se entiende como un sistema de integración humana, renovación orgánica, reeducación afectiva y reaprendizaje de las funciones originarias de la vida (Toro, 2009). El método Biodanza fue creado por Rolando Toro, psicólogo chileno, tras haber experimentado los efectos beneficiosos de la música y la danza en el bienestar de pacientes con problemas de salud mental (Cavalcante y Wagner, 2015).

Posteriormente, este sistema tuvo reconocimiento internacional y fue introducido en Europa en los años 80. Este

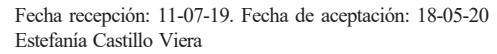

método propone la integración vivencial mediante la música, el canto y la danza tanto a nivel individual como grupal, estimulando la parte sana de las personas (Stueck, Villegas, Bauer, Terren, Toro, y Sack, 2009).

Es un método de toma de conciencia del momento presente, que implica funciones orgánicas, emocionales y de pensamiento, proporcionando una experiencia subjetiva, basada en la conexión con la vida y sus experiencias placenteras (Toro, 2009).

La vivencia es el elemento fundamental y que da eficacia a esta disciplina, y tiene como objetivo facilitar la conexión y la expresión de las emociones, y se propone como un mecanismo capaz de modificar el organismo y la existencia en los niveles afectivo-motor y existencial (Pessoa Santos, 2010). El movimiento va acompañado de música seleccionada en base a sus contenidos emocionales, y el grupo afectivamente integrado ofrece posibilidades diversificadas de comunicación y actúa como continente afectivo para favorecer la expresión de las emociones (Toro, 2009).

Los efectos de Biodanza serían de naturaleza diversa, y autores como Toro (2009) o Pessoa Santos (2010), le han atribuido beneficios dopaminérgicos causantes de buen humor; estimulación de las endorfinas, serotonina y acetilcolina que se manifiestan en las sensaciones de placer; aumenta la producción de GABA y ácido glutámico que producen sensación de tranquilidad; activa los circuitos neurológicos del sistema límbico-hipotalámico que ponen 
en marcha a su vez las glándulas endocrinas asociadas y por lo tanto el sistema inmunológico, recuperándose así el equilibrio psicosomático.

Biodanza actúa induciendo sistemática y regularmente vivencias partiendo de la danza (García, 2008). Los ejercicios que se plantean responden al objetivo de integración a distintos niveles: grupal, integración motora, sensitivo-motora, afectivo-motora y cognitivo-motora, cada una con unas características específicas, y con un tipo de movimiento asociado (Pessoa Santos, 2010). Según aumenta el tiempo de práctica se van proponiendo nuevos ejercicios para la profundización del método, a la vez que se introducen ejercicios de mayor contacto (García, 2008). Los cambios que se producen en los participantes tienden a incorporarse en su estilo de vida, se denominan de primer o segundo orden, en función del grado de adquisición de los mismos (García, 2008).

Bajo la pregunta de investigación ¿puede la biodanza contribuir a la mejora de la salud y el bienestar? pretendemos analizar las evidencias científicas que arrojan datos al respecto. Por esto, el objetivo de este artículo fue realizar una revisión sistemática de la literatura científica sobre biodanza y su relación con la salud.

\section{Método}

Esta revisión de la literatura sobre biodanza y salud se llevó a cabo siguiendo la guía práctica de la declaración PRISMA para revisiones sistemáticas (Urritia y Bonfill, 2010; Moher et al, 2015).

\section{Criterios de inclusión y exclusión}

Los criterios de inclusión fueron: artículos a texto completo; en lengua inglesa o castellana; que analizan la relación entre biodanza y variables de salud y calidad de vida.

Los criterios de exclusión fueron: trabajos de revisión teórica, trabajos de revisión sistemática, no tener un diseño experimental o cuasi experimental; no estar relacionado con el tema de estudio.

\section{Estrategia de búsqueda y selección de estudios}

Las bases de datos sobre las que se realizaron la búsqueda fueron la base de datos de Web of Science y Scopus, durante los meses de noviembre y diciembre de 2019, sin límite temporal. La estrategia de búsqueda fue: (biodanza OR biodance)AND (health OR «life satisfaction» OR well-being OR self-esteem OR stress OR depression OR «quality of life»).

El procedimiento seguido fue: búsqueda en las bases de datos en función de los términos de búsqueda; lectura del título y resumen para localizar a aquellos que inicialmente cumplían los criterios de inclusión. Posteriormente se leyeron a texto completo y se excluyeron aquellos que no cumplían todos los requisitos de inclusión

Extracción de datos y proceso de selección de estudios

Finalmente, se extrajeron los datos de los artículos seleccionados utilizando una plantilla diseñada previamente, incluyendo: diseño, población y muestra, país, instrumentos, beneficios de biodanza, y calidad del estudio para facilitar la simplificación de los datos y su análisis. Estos datos fueron extraídos por un autor, y verificados juntos con los otros autores para el análisis de calidad de los artículos extraídos.

\section{Estudio cualitativo y riesgo de sesgo}

El riesgo de sesgo fue evaluado utilizando el Quality Assessment Tool for Quantitative Studies 10 (2010).

La hoja de evaluación de calidad comprendida por 19 ítems evalúa los siguientes elementos: el riesgo de sesgo de selección; características del diseño del estudio; manejo de los factores de confusión; manejo del enmascaramiento; métodos de recolección de los datos; abandono del estudio; integridad de la intervención; análisis realizado. Cada apartado fue clasificado como fuerte, moderado o débil en cuanto a la calidad metodológica. Se determinó una calificación global basada en las puntuaciones de cada componente. Dos investigadores calificaron los artículos y las discrepancias se resolvieron mediante acuerdo. No se excluyeron los estudios por calidad metodológica.

\section{Resultados}

En el diagrama de flujo representado en la figura 1 se muestran los resultados obtenidos tras la revisión sistemática. La primera búsqueda presentó 70 registros. Después de excluir los duplicados quedaron 38 registros que, tras aplicarse los criterios de inclusión y exclusión durante la fase de cribado y posterior elegibilidad, quedaron reducidos a 12 estudios. Merece la pena destacar que de los 16 registros excluidos a texto completo la gran mayoría (11) lo fueron por tratarse de revisiones teóricas.

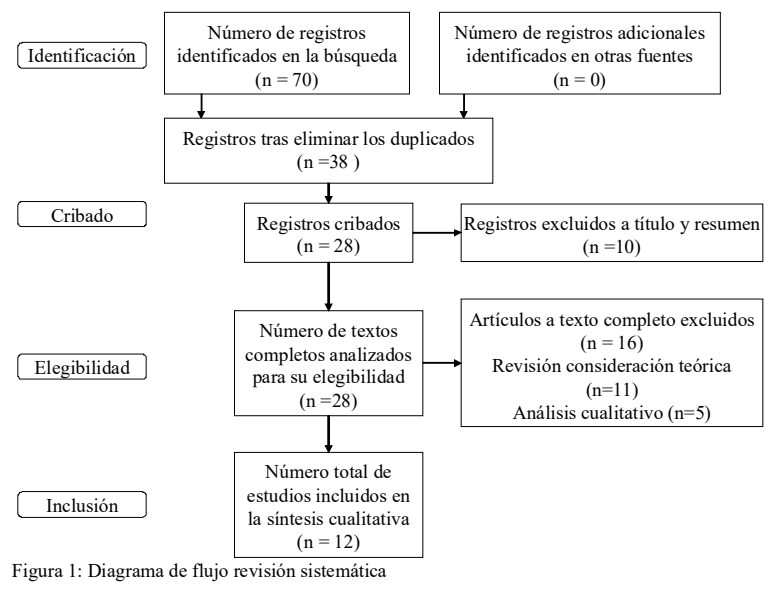

De los doce estudios resultantes de la fase final de cribado y elegibilidad, reflejados con detalle en la tabla 1, la mitad de ellos (5) se realizaron con mujeres maduras diagnosticadas de fibromialgia (Abad, Castillo y Orizia, 2013; Carbonel, Aparicio, Martins, Gatto-Cardia, Ortega ,Tercedor, Ruiz y Delgado, 2010; Carbonel, Ruiz, Aparicio, Martins, GattoCardia, Martínez, Ortega y Delgado, 2012; López, Castro, Fernández, Matarán y Rodríguez, 2012; Segura, Gatto-Cardia, Martins, Delgado, Aparicio y Carbonel-Baeza, 2017) o en general con pacientes de fibromialgia (López, Fernández, Matarán, Rodriguez y Aguilar, 2013); Igualmente, cuatro de estos estudios cuentan con una muestra compuesta por población menor de edad, uno realizado con niñas en edad 
preescolar (Stueck, Villegas, Lahn, Bauer, Toft y Sacks, 2016), dos realizados con niños y niñas escolarizados en la educación primaria (Castillo, Inostroza, Muñoz y Toro, 2019; Hernández, Fierro, Fernández y Sáenz, 2018) y un último sobre población adolescente en la enseñanza secundaria (Rosa, Ascione y Di Palma, 2019). Finalmente, otros dos estudios (López, Baldrich, Ruíz, Cortés, Lopezosa-Estepa y Román, 2017) se han realizado contando con población universitaria. Muy significativamente, 8 de los 12 estudios considerados han sido realizados en España.

Sobre las variables de estudio, las más comunes son el estudio de la depresión y el afrontamiento y manejo del dolor, apareciendo conjuntamente hasta en cinco estudios de los 12 seleccionados. La inteligencia emocional se analizó también en tres de los estudios, seguida de la identificación y medición de otras variables psicológicas tales como la ansiedad, la autoeficacia, la autoestima, la satisfacción con la vida y el impacto de la fibromialgia, la calidad del sueño, la habilidad para expresión emocional o la concentración. Las variables físico-corporales como el porcentaje de grasa corporal, la agilidad, la composición corporal o el consumo máximo de oxígeno son también frecuentes y están presentes en cuatro de estos estudios. Todos los estudios fueron de corte cuantitativo, y diseño cuasi experimental o ensayo clínico.

\section{Discusión}

Después de analizar los textos encontrados se agruparon los resultados según los efectos identificables de la Biodanza en las principales áreas temáticas que han orientado la revisión. Si bien se evidencian hallazgos positivos en el ámbito de la salud y el bienestar humano, apareciendo Biodanza como un recurso emergente valioso en la prevención y cuidado de la salud, es importante tener una visión crítica de los resultados a la luz del limitado número de artículos En concreto, se identifican las siguientes evidencias:

Tabla 1

\begin{tabular}{|c|c|c|c|}
\hline AUTOR/ES & AÑO & MUESTRA & PAÍS \\
\hline $\begin{array}{l}\text { Abad Robles, Castillo Viera } \\
\text { y Orizia Pérez }\end{array}$ & 2013 & $\begin{array}{l}\text { Mujeres Mayores } \\
\text { de } 40 \text { años } \\
n=35\end{array}$ & España \\
\hline $\begin{array}{l}\text { Carbonell Baeza, Aparicio, } \\
\text { Martins Pereira, Gatto- } \\
\text { Cardia,Ortega,Huertas,Terc } \\
\text { edor, Ruiz, Delgado } \\
\text { Fernandez }\end{array}$ & 2010 & $\begin{array}{l}\text { Mujeres con } \\
\text { fibromialgia } \\
\mathrm{n}=59\end{array}$ & España \\
\hline $\begin{array}{l}\text { Carbonell Baeza, Ruiz, } \\
\text { Aparicio, Martins Pereira, } \\
\text { Gatto-Cardia, Martínez, }\end{array}$ & 2012 & $\begin{array}{l}\text { Mujeres con } \\
\text { fibromialgia } \\
\mathrm{n}=38\end{array}$ & España \\
\hline
\end{tabular}

Vítica DISEÑO Inteligencia Emocional Percibida $\quad$ Cuasiexperimental $\quad$ la práctica de biodanza de forma regular mejora el grado de (percepción, comprensión y con grupo control inteligencia emocional de las practicantes y muy especialmente Umbral de dolor; Composición $\quad$ Ensayo controlado $\quad$ La intervención con biodanza reduce el impacto del dolor en corporal; Estado físico; Variables pre test y re test con grupo mujeres con fibromialgia. Los resultados muestran que esta psicológicas (impacto de la control intervención puede ser un valioso recurso en un corto plazo fibromialgia, salud, gestión del dolor, biodanza durante 3 meses $\quad$ para manejar el dolor. ansiedad y depresión, autoestima y utoeficacia general).

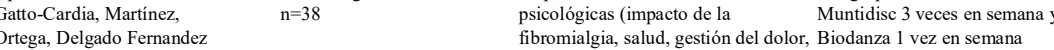
ansiedad y depresión, autoestima y autoeficacia general) sociales y el uso de estrategias pasivas de afrontamiento en mujeres con fibromialgia; también se obtuvieron beneficios adicionales a través de esta intervención multidisciplinar relativos a la depresión y la fatiga. En ambos grupos registraron mejoras en la medición del impacto de la fibromialgia (FIQ), si bien no está claro que se deban a las intervenciones realizadas.

\begin{tabular}{|c|c|c|c|c|c|c|}
\hline \multirow{2}{*}{\multicolumn{2}{|c|}{$\begin{array}{l}\text { Castillo; Inostroza; Muñoz; } 2019 \\
\text { Toro }\end{array}$}} & \multirow{3}{*}{$\begin{array}{l}\begin{array}{l}\text { Niños y niñas. } \\
\mathrm{n}=54\end{array} \\
\begin{array}{l}\text { Niños y } \mathrm{ni} \\
\text { ñas } \\
\mathrm{n}=53\end{array}\end{array}$} & \multirow{3}{*}{$\begin{array}{l}\text { Chile } \\
\text { España }\end{array}$} & \multirow{3}{*}{$\begin{array}{l}\text { Autoestima } \\
\begin{array}{l}\text { Autoestima; Inteligencia emocional; } \\
\text { Satisfacción con la vida; Consumo } \\
\text { máximo de oxígeno; Agilidad. }\end{array}\end{array}$} & \multirow{3}{*}{$\begin{array}{l}\text { Estudio experimental con grupo } \\
\text { experimental y } \\
\text { de control }\end{array}$} & \multirow[b]{2}{*}{$\begin{array}{l}\text { intervenciones realizadas. } \\
\text { Tras una intervención grupal basada en el sistema Biodanza se } \\
\text { constata un aumento significativo de la puntuación media } \\
\text { referida a la autoestima escolar en el grupo experimental, } \\
\text { generándose cambios positivos en el grupo de estudiantes } \\
\text { intervenido. }\end{array}$} \\
\hline & & & & & & \\
\hline $\begin{array}{l}\text { Hernández López, Fierro } \\
\text { Suero, Fernández Ozcorta, } \\
\text { Sáenz López Buñuel }\end{array}$ & 2018 & & & & & $\begin{array}{l}\text { El programa Biodanza en escolares conduce a mejoras } \\
\text { significativas en la autoestima, inteligencia emocional y } \\
\text { agilidad. Para consumo máximo de oxígeno y satisfacción con } \\
\text { la vida, se han encontrado mejoras, pero no son significativas. }\end{array}$ \\
\hline $\begin{array}{l}\text { López Rodriguez, Baldrich } \\
\text { Rodriguez, Ruíz Muelle, } \\
\text { Cortés Rodriguez, } \\
\text { Lopezosa Estepa, Román }\end{array}$ & 2017 & $\begin{array}{l}\text { Estudiantes } \\
\text { Universitarios } \\
\text { con estrés } \\
\text { percibido } \\
\mathrm{n}=121\end{array}$ & España & $\begin{array}{l}\text { Depresión; Estrés percibido; Calidad } \\
\text { del sueño. }\end{array}$ & $\begin{array}{l}\text { Ensayo controlado } \\
\text { aleatorio. Gpo. Exp. una clase } \\
\text { semanal de biodanza 90min. } \\
\text { Gpo control: lista } \\
\text { espera. }\end{array}$ & $\begin{array}{l}\text { Biodanza es un medio efectivo para prevenir y manejar } \\
\text { problemas en estudiantes universitarios, mostrando resultados } \\
\text { positivos en el estrés percibido y en la depresión en jóvenes } \\
\text { adultos. }\end{array}$ \\
\hline $\begin{array}{l}\text { López Rodriguez, Castro } \\
\text { Sánchez, Fernández } \\
\text { Martinez, Matarán } \\
\text { Peñarrocha, Rodríguez } \\
\text { Ferrer. }\end{array}$ & 2012 & $\begin{array}{l}\text { Mujeres con } \\
\text { fibromialgia } \\
\mathrm{n}=39\end{array}$ & España & $\begin{array}{l}\text { Calidad del sueño; Ansiedad; } \\
\text { Depresión; Dolor; Calidad de vida } \\
\text { (Impacto de la fibriomialgia). }\end{array}$ & $\begin{array}{l}\text { Ensayo clínico aleatorio en } \\
\text { ámbito comunitario. } 2 \text { gpos. } \\
\text { Interv. }\end{array}$ & $\begin{array}{l}\text { El tratamiento mediante un programa de Biodanza acuática } \\
\text { produce mejoras en la calidad del sueño, ansiedad e impacto de } \\
\text { fibromialgia, así como en el dolor y puntos dolorosos en las } \\
\text { mujeres con fibromialgia. }\end{array}$ \\
\hline $\begin{array}{l}\text { López Rodriguez, } \\
\text { Fernández Martínez, } \\
\text { Matarán } \\
\text { Peñarrocha, Rodriguez } \\
\text { Ferrer, Aguilar Ferrándiz }\end{array}$ & 2013 & $\begin{array}{l}\text { Pacientes con } \\
\text { fibromialgia } \\
\mathrm{n}=59\end{array}$ & España & $\begin{array}{l}\text { Dolor; Impacto de la fibromialgia; } \\
\text { Depresión }\end{array}$ & $\begin{array}{l}\text { Estudio experimental con gpo } \\
\text { experimental (biodanza } \\
\text { acuática) y } \\
\text { Gpo control (stretching) }\end{array}$ & $\begin{array}{l}\text { El tratamiento mediante un programa de Biodanza acuática } \\
\text { produce mejoras en el dolor así como en el impacto de la } \\
\text { fibromialgia y la depresión. }\end{array}$ \\
\hline Rosa; Ascione; Di Palma & 2019 & $\begin{array}{l}\text { Estudiantes de } \\
\text { Educación } \\
\text { Secundaria } \\
\mathrm{n}=16\end{array}$ & Italia & $\begin{array}{l}\text { Conciencia corporal; Habilidad para } \\
\text { la expression corporal; Control } \\
\text { motor; Orientación especial; } \\
\text { Conocimiento y respeto de normas } \\
\text { del juego; Conocimiento conceptual } \\
\text { relacionado con el bienestar } \\
\text { psicosocial. }\end{array}$ & control & $\begin{array}{l}\text { La inclusión de Biodanza junto a las propuestas didácticas } \\
\text { tradicionales en el campo de enseñanza deportiva se ha } \\
\text { mostrado efectiva desde una perspectiva pedagógica, existiendo } \\
\text { evidencias de mejora de rendimiento en el entrenamiento de } \\
\text { ambos grupos (control y experimental). }\end{array}$ \\
\hline $\begin{array}{l}\text { Segura Jiménez, Gatto- } \\
\text { Cardia, Martins Pereira, } \\
\text { Delgado Fernandez, } \\
\text { Aparicio, Carbonel-Baeza }\end{array}$ & 2017 & $\begin{array}{l}\text { Mujeres } \\
\text { Con fibromialgia } \\
\mathrm{n}=27\end{array}$ & España & $\begin{array}{l}\text { Severidad del dolor; Porcentaje de } \\
\text { grasa corporal; Satisfacción con las } \\
\text { sesiones. }\end{array}$ & $\begin{array}{l}\text { Diseño cuasi- } \\
\text { experimental }\end{array}$ & $\begin{array}{l}\text { Una intervención de Biodanza redujo la gravedad del dolor } \\
\text { agudo en mujeres con fibromialgia. Los beneficios de la } \\
\text { gravedad del dolor después de la sesión de Biodanza fueron } \\
\text { mayores a medida que avanzaba la intervención. El presente } \\
\text { estudio también resaltó la importancia de mantener un bajo } \\
\text { porcentaje de grasa corporal y asistir a las sesiones en aquellos } \\
\text { días en que el dolor es más intenso, ya que los beneficios } \\
\text { pueden ser incrementales. Además, la satisfacción con la sesión } \\
\text { fue otro aspecto importante relacionado con la reducción. }\end{array}$ \\
\hline $\begin{array}{l}\text { Stueck, Villegas, Lahn, } \\
\text { Bauer, Toft y Sacks }\end{array}$ & 2016 & $\begin{array}{l}\text { Niñas de Jardín } \\
\text { de Infancia } \\
\mathrm{n}=10\end{array}$ & Alemania & $\begin{array}{l}\text { Niveles de cortisol; Parámetros } \\
\text { cognitivos (reconocimiento } \\
\text { emocional y concentración) }\end{array}$ & & $\begin{array}{l}\text { Es posible que TANZPRO Biodanza tenga un efecto positivo a } \\
\text { corto plazo en la capacidad de regulación automática de niños } \\
\text { de } 4 \text { a } 6 \text { años (medido por la modulación mejorada del cortisol). } \\
\text { Esta intervención podría permitir el desarrollo de habilidades } \\
\text { sociales como la capacidad de reconocimiento de emociones; }\end{array}$ \\
\hline $\begin{array}{l}\text { Stueck; Kaloet; Villegas; } \\
\text { Utami }\end{array}$ & 2019 & $\begin{array}{l}\text { Adultos } \\
\text { (población } \\
\text { universitaria) } \\
\mathrm{n}=34\end{array}$ & Indonesia & $\begin{array}{l}\text { Habilidad para la expresión } \\
\text { emocional; Habilidad para la } \\
\text { expresión de necesidades. }\end{array}$ & $\begin{array}{l}\text { Comparación entre dos grupos: } \\
\text { Grupo } 1 \text { (Biodanza); Grupo } 2 \\
\text { (Soe Verbal) }\end{array}$ & $\begin{array}{l}\text { Biodanza mejora el reconocimiento emocional y la expresión } \\
\text { emocional, no así la expresión de necesidades. En el grupo de } \\
\text { practicantes de Biodanza son más frecuentes los individuos que } \\
\text { consiguen satisfacer necesidades psicológicas y expresar } \\
\text { necesidades fisicas, en comparación con los practicantes de SoE } \\
\text { (School of Empathy). }\end{array}$ \\
\hline
\end{tabular}




\section{Depresión y afrontamiento del dolor}

Dado que el estudio de las propiedades de la Biodanza parece haber estado concentrado en pacientes de fibromialgia, buena parte de sus resultados constatables están ligados al manejo del dolor y el impacto de la enfermedad en la calidad de vida de las pacientes. En este sentido, la práctica de Biodanza mejora el índice de dolor, reduce el impacto de la fibromialgia en las actividades de la vida cotidiana e igualmente el de la depresión (Carbonell-Baeza et al., 2010; López, Fernández, Matarán, Rodriguez y Ferrándiz, 2013). Produce una reducción acumulada de la gravedad del dolor (Segura et al, 2017) y mejora la composición corporal (Carbonell-Baeza et al, 2010). También favorece una mejora significativa en algunos de los principales síntomas de los pacientes de fibromialgia, ya que aporta a la recuperación de la calidad del sueño, reduce el estado de ansiedad y promueve una disminución de la sintomatología y una mayor autonomía, lo cual propicia un aumento de la calidad de vida (López, Castro, Fernández, Matarán y Rodríguez, 2012). En definitiva, parece claro que, en líneas generales, promueve la salud física y mental para la mejora de la calidad de vida en personas con fibromialgia, si bien se ha encontrado también un único caso que evidencia que la Biodanza no ha mostrado mayores beneficios para las pacientes que una intervención de corte multidisciplinar (Carbonel et al., 2012).

\section{Efectos sobre la autoestima y expresión de las emocio- nes}

Parecen evidentes los efectos sobre la expresión y regulación emocional, así como sobre la autoestima. La Biodanza tiene un efecto específico del estado de ánimo hacia la alegría, consigue que las personas realicen una descripción más diferenciada de sus sentimientos, al tiempo que aumenta la expresión de las emociones, modulación y equilibrio emocional (Stuek y Tofts, 2016). Con su práctica, parece permitirse el desarrollo de habilidades sociales como la capacidad de reconocimiento de emociones, así como la mejora del reconocimiento y la expresión emocional tanto en niños como en la población adulta (Stueck, Villegas, Lahn, Bauer, Toft y Sacks, 2016; Stueck, Kaloet, Villegas y Utami, 2019). Otros estudios han encontrado que se produce un aumento significativo en los niveles de inteligencia emocional percibida, especialmente en la dimensión de comprensión de las propias emociones, seguida de la regulación y finalmente la percepción (Abad, Castillo y Orizia, 2013). También se ha comprobado en población infantil escolarizada una mejora significativa en la autoestima e inteligencia emocional (Castillo, Inostroza, Muñozy Toro, 2019; Hernández, Fierro, Fernández y Sáenz, 2018). A tenor de los resultados identificados por la revisión, la Biodanza tiene también un efecto positivo sobre la reducción del estrés percibido, como se ha constatado también en estudiantes universitarios (López, Baldrich, Ruíz, Cortés, Lopezosa y Román, 2017).

\section{Conclusiones}

El objetivo de este estudio fue realizar una revisión sistemática de la literatura científica sobre biodanza y su relación con la salud. El escaso número de publicaciones científicas sobre el tema dificulta generalizar los resultados y beneficios de Biodanza en el bienestar que son enunciados en los textos analizados. Estas circunstancias motivan la realización de nuevas investigaciones que permitan validar la eficacia de este prometedor método en el área del bienestar subjetivo. Por otro lado, El itinerario de mejora para estos nuevos estudios de investigación deberá incluir el uso de grupos de control, el empleo de escalas homologadas científicamente, períodos de intervención más prolongados, pruebas de estabilización de los efectos después de un tiempo de realizados los estudios.

Este texto supone el punto de partida para generar conocimiento científico sobre esta técnica corporal emergente, potencialmente un importante instrumento de trabajo comunitario y promoción de la salud al alcance de cualquier persona.

\section{Referencias}

Abad, M. T., Castillo, E., \& Orizia, A. C. (2014). Los efectos de un programa motor basado en la biodanza en relación con parámetros de inteligencia emocional en mujeres. Cuadernos De Psicología Del Deporte, 14(1), 13-22. doi: 10.4321/S1578-84232014000100002.

Carbonell-Baeza, A., Aparicio, V. A., Martins-Pereira, C. M., Gatto-Cardia, C. M., Ortega, F. B., Huertas, F. J., ... Delgado-Fernandez, M. (2010). Efficacy of Biodanza for Treating Women with Fibromyalgia. The Journal of Alternative and Complementary Medicine, 16(11), 11911200. doi:10.1089/acm.2010-0039

Carbonell-Baeza, A., Ruiz, J. R., Aparicio, V. A., MartinsPereira, C., Gatto-Cardia, M., Martinez, J. M., DelgadoFernandez, M. (2012). Multidisciplinary and biodanza intervention for the management of fibromyalgia. Acta Reumatologica Portuguesa, 37(3), 240-250.

Castañeda, G. M. (2009). La biodanza como práctica corporal en relación con la promoción de la salud. Educación Física y deporte, 28, 81-90.

Castillo, F., Inostroza, K., Munoz, B., Toro, M. (2019) Biodanza and Physical Education: Disciplinary configuration to increase school's self-esteem. VIREF-Revista de Educación Física. 8(3),19-32.

Cavalcante, R., Wagner C. (2015). Educaçao Biocéntrica: ciencia, arte, mística, amor y transformaçao. Ceará, Brasil: Universidade Estadual Vale do Acaraú (UVA)

García, C. (2008). Biodanza: el arte de danzar la vida. La vivencia como terapia. Buenos Aires: Ediciones Pausa.

Giannelli, M.T., Giannino, P., Mengarelli, A(2015). Efficacia sulla salute di un corso annuale di Biodanza: Uno studio empirico. Psicologia della Salute (1) 84-107.

Hernández López, J. R., Fierro Suero, S., Fernández-Ozcorta, E., \& Sáenz-López, P. (2018). Efectos de un programa de biodanza en relación a parámetros físicos y psicológicos en educación primaria. e-balonmano.com: Revista de Ciencias del Deporte, 14(1), 55-64.

León, P., \& González-Martí, I. (2017). Efectos de la biodanza sobre la afectividad y espontaneidad en niños.

López-Rodríguez, M. del M., Castro-Sánchez, A. M., Fernández-Martínez, M., Matarán-Peñarrocha, G. A., \& Rodríguez-Ferrer, M. E. (2012). Comparación entre biodanza en medio acuático y stretching en la mejora de 
la calidad de vida y dolor en los pacientes con fibromialgia. Atención Primaria, 44(11), 641-649. doi: 10.1016/ j.aprim.2012.03.002

López-Rodríguez, M. M., Baldrich-Rodríguez, I., Ruiz-Muelle, A., Cortés-Rodríguez, A. E., Lopezosa-Estepa, T., \& Roman, P. (2017). Effects of Biodanza on Stress, Depression, and Sleep Quality in University Students. The Journal of Alternative and Complementary Medicine, 23(7), 558-565. doi:10.1089/acm.2016.0365

López-Rodríguez, M. M., Fernández-Martínez, M., MataránPeñarrocha, G. A., Rodríguez-Ferrer, M. E., Granados Gámez, G, \&AguilarFerrándiz, E. (2013). Efectividad de la biodanza acuática sobre la calidad del sueño, la ansiedad $\mathrm{y}$ otros síntomas en pacientes con fibromialgia. Medicina Clínica, 141(11), 471-478. doi: 10.1016/ j.medcli.2012.09.036

Moher, D., Shamseer, L., Clarke, M., Ghersi, D., Liberati, A., ... Stewart, L. A. (2015). Preferred reporting items for systematic review and meta-analysis protocols (PRISMA-P) 2015 statement. Systematic Reviews, 4(1). doi:10.1186/2046-4053-4-1

Padilla Moledo, C., \& Coterón López, J. (2013). ¿Podemos mejorar nuestra salud mental a través de la Danza?: una revisión sistemática. Retos, 24, 194-197.

Pessoa Santos, M. L. (2010). Biodanza: Vida y Plenitud (1st ed.). Belo Horizonte, Brasil.

Rosa, R., Ascione, A., Di Palma, D. (2019) Biodanza laboratory And Exercise, 14, 169-177. https://doi.org/10.14198/ jhse.2019.14.Proc2.03

Segura-Jiménez, V., Gatto-Cardia, C. M., Martins-Pereira, C. M., Delgado-Fernández, M.,Aparicio, V.A., \& CarbonellBaeza, A. (2017). Biodanza Reduces Acute Pain Severity and experimental pedagogy. Journal Of Human Sport

in Women with Fibromyalgia. Pain Management Nursing, 18(5), 318-327. doi: 10.1016/j.pmn.2017.03.007

Stueck, M., \& Tofts, P. S. (2016). Biodanza Effects on Stress Reduction and Well-Being-A Review of Study Quality and Outcome. Signum Temporis, 8(1), 57-66. doi: 10.1515/ sigtem-2016-0018

Stueck, M., Villegas, A., Bauer, K., Terren, R., Toro, V., \& Sack, U. (2009). Psycho-Immunological Process Evaluation of Biodanza. Journal of Pedagogy and Psychology «Signum Temporis», 2(1). doi:10.2478/v10195-011-00247

Stueck, M., Villegas, A., Lahn, F., Bauer, K., Tofts, P., \& Sack, U. (2016). Biodanza for kindergarten children (TANZPROBiodanza): reporting on changes of cortisol levels and emotion recognition. Body, Movement and Dance in Psychotherapy, 11(1), 75-89. doi:10.1080/ 17432979.2015.1124923

Stueck, M., Kaloeti, Dian, S., Villegas, A., Utami, D. S., The influence of Biodanza and School of Empathy verbal respectful communication on the ability to express emotions and needs: a pilot study among adults in Indonesia. Health Psychology Report, 7(4), 334 -340. https://doi.org/10.5114/hpr.2019.88665

Thomas, B.H.; Ciliscka, D.; Dobbins, M., \& Micucci, S. (2004). A process for systematically reviewing the literature: providing the research evidence for public helath nursing interventions. Worldviews on Evidence-Based Nursing. 2004, 176-184

Toro, R. (2009). Biodanza (3rd ed.). Santiago, Chile: Cuarto Propio.

Urrútia, G; Bonfill, X. (2010). Declaración PRISMA: una propuesta para mejorar la publicación de revisiones sistemáticas y metaanálisis. Med Clin (Barc). 2010;135(11):507-511.

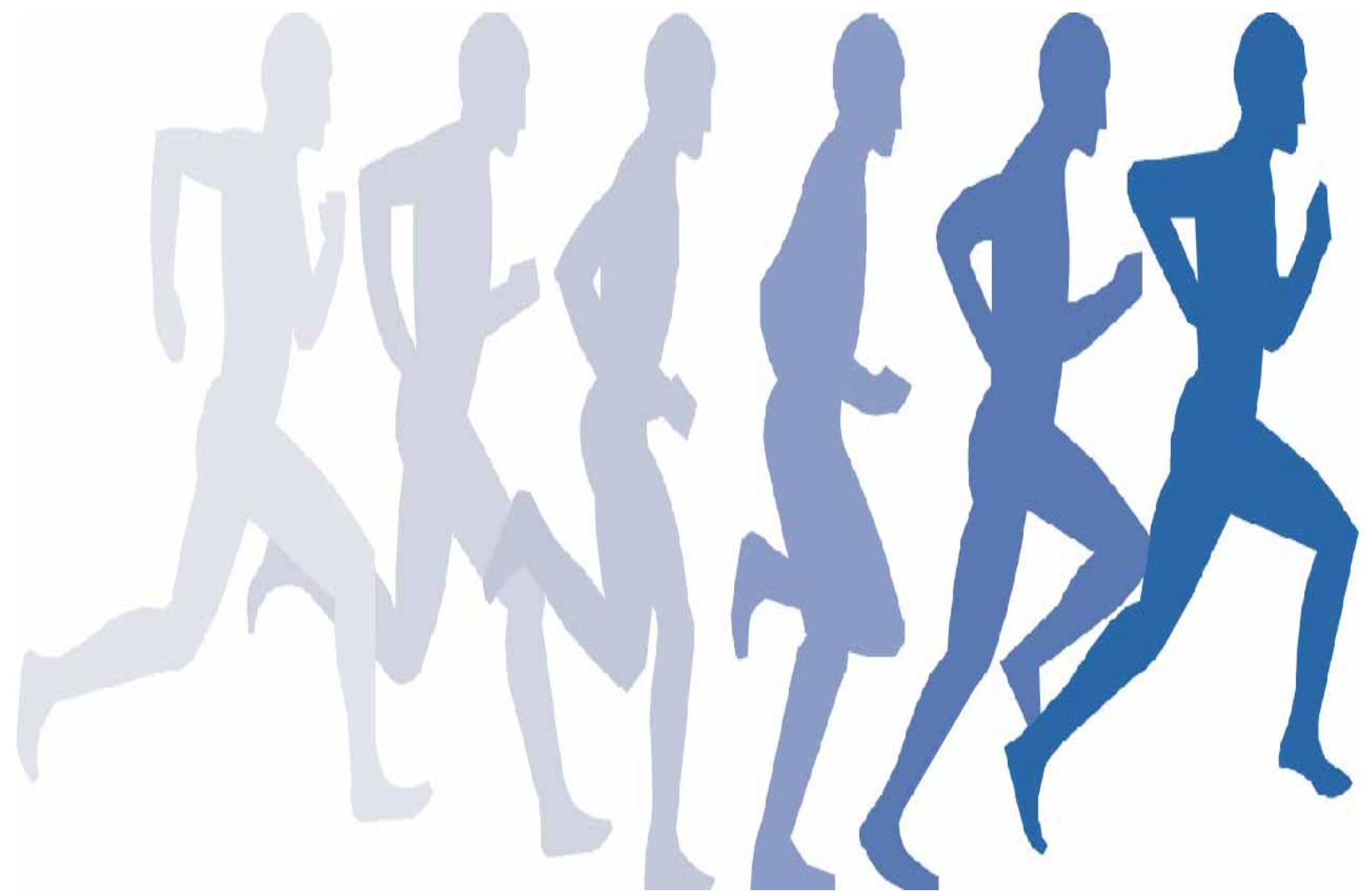

